\title{
A Generalization OF A WAITING TIME PROBLem
}

\author{
B. S. EL-DESOUKY* AND \\ S. A. HUSSEN, ${ }^{*}$ Faculty of Science, Aswan
}

\begin{abstract}
An urn contains $m$ types of balls of unequal numbers. Let $n_{i}$ be the number of balls of type $i, i=1,2, \cdots, m$. Balls are drawn with replacement until first duplication. In the case of finite memory of order $k$, the distribution of $Y_{m, k}$, the number of drawings required, is discussed. Special cases are obtained.
\end{abstract}

\section{Introduction}

An urn contains $m$ distinguishable balls which are sampled one at a time with replacement. The sampling is continued until the first duplication. Let $X_{m}$ be the number of drawings required.

This problem, which was solved by McCabe [4], is a special case of the problem of waiting time until first duplication with finite memory of order $k$ (Arnold [1]), in which sampling is continued until a ball is drawn to duplicate one of the $k$ preceding balls drawn. Let $X_{m k}$ be the number of draws required when there are $m$ balls in the urn and there is finite memory of order $k$. The distribution of $X_{m k}$ is found by Arnold [1].

In this paper we consider two cases.

Case 1. We generalize McCabe [4] as follows. Suppose that we have an urn containing $m$ types of balls with $n_{i}$ the number of balls of type $i, i=1,2, \cdots, m$. Assume that balls are sampled one at a time with replacement and the sampling is continued until the first duplication (i.e., until a ball of the same type has been drawn twice), and $Y_{m}$ is the number of drawings performed.

Case 2. Case 1 can be considered as a special case of the problem of waiting time until first duplication with finite memory of order $k$.

In this case sampling is continued until a ball is drawn to duplicate one of the $k$ immediately preceding balls (one of each type) drawn. For example, when $k=1$, sampling stops only when two successive drawings yield a ball of the same type.

Let $Y_{m, k}$ be the number of draws required when there are $m$ types of balls of unequal numbers and there is a finite memory of order $k$, which can be considered as a generalization of Arnold's problem [1]. It is clear that $Y_{m}$ is identical with $Y_{m, m}$ and if $k>m$, then $Y_{m, k}$ also has the same distribution as $Y_{m, m}$.

\section{The distribution of $Y_{m, k}$}

If $k=m$ it is clear that the random variable $Y_{m, m}$ cannot be smaller than 2 or larger than $m+1$. When $k<m$, the random variable $Y_{m, k}$ may assume any value greater than or equal to 2. Hence we discuss the two cases. First we consider the distribution of $Y_{m, k}$ (Case 2) which gives us the distribution of $Y_{m, m}$ (Case 1) as a special case.

If $k<m$, for any integer $j$ satisfying $1 \leqq \leqq k+1$,

$$
\begin{gathered}
P\left(Y_{m, k}>j\right)=P \text { (the first } j \text { balls are all distinct, one of each type) } \\
P\left(Y_{m, k}>j\right)=\sum_{\pi} p_{\pi_{(1)}} p_{\pi_{(2)}} \cdots p_{\pi_{(j)}},
\end{gathered}
$$

Received 18 September 1989; revision received 25 May 1990.

* Postal address for both authors: Department of Mathematics, Faculty of Science, Aswan, Egypt. 
where the summation is over all permutations $\left(\pi_{(1)}, \pi_{(2)}, \cdots, \pi_{(j)}\right)$ of the integers $1,2, \cdots, m$ taken $j$ at a time, and $p_{i}=n_{i} / N, N=\sum_{i=1}^{m} n_{i}$, is the probability that a ball of type $i$ is drawn.

In the special case $k=m$, this yields the following result.

Theorem 2.1. The generating function of the probabilities $P\left(Y_{m, m}>j\right), j=1,2, \cdots, m$ is given by

$$
\sum_{j=0}^{m} P\left(Y_{m, m}>j\right) \frac{t^{j}}{N^{j}}=\prod_{i=1}^{m}\left(1+p_{i} t\right)
$$

Proof. Note that

$$
\sum_{\pi} p_{\pi_{(1)}} p_{\pi_{(2)}} \cdots p_{\pi_{(j)}}=j ! \sum_{c(j)}\left[\prod_{k=1}^{j} p_{i_{k}}\right]
$$

where the second summation is over all unordered subsets $\left(i_{1}, i_{2}, \cdots, i_{j}\right)$ of size $j$ of the integers $1,2, \cdots, m$. But the coefficient of $t^{j}$ on the right-hand side of (2.2) is clearly

$$
\sum_{c(j)}\left[\prod_{i=1}^{j} p_{i_{k}}\right]
$$

hence the theorem is proved.

From (2.2)

$$
\begin{aligned}
\sum_{j=0}^{m} P\left(Y_{m, m}>j\right) \frac{t^{j}}{j !} & =\left(1+n_{1} \frac{t}{N}\right)\left(1+n_{2} \frac{t}{N}\right) \cdots\left(1+n_{m} \frac{t}{N}\right), \quad N=\sum_{i=1}^{m} n_{i} \\
& =\sum_{i=0}^{m} S_{n}(m, m-i)\left(\frac{t}{N}\right)^{i},
\end{aligned}
$$

hence

$$
P\left(Y_{m, m}>j\right)=\frac{j !}{N^{j}} S_{n}(m, m-j)=\frac{(-1)^{j} j !}{N^{j}} s_{n}(m, m-j),
$$

where $S_{n}(m, k)$ is the unsigned generalized Stirling number of the first kind and $S_{n}(m, k)=$ $(-1)^{m-k} s_{n}(m, k)$ and $s_{n}(m, k)$ is the generalized Stirling number of the first kind associated with the real numbers $n_{1}, n_{2}, \cdots, n_{m}$ (see [2] and [3]).

If $n_{i}=i, i=1,2, \cdots, m$, then

$$
P\left(Y_{m, m}>j\right)=\frac{(-1)^{j} j !}{N^{j}} s(m+1, m+1-j), \quad N=m(m+1) / 2,
$$

where $s(m, k)$ is the Stirling number of the first kind.

If $n_{i}=n, i=1,2, \cdots, m$, i.e. there is an equal number of balls from each type, then

hence

$$
\sum_{j=0}^{m} P\left(Y_{m, m}>j\right) \frac{t^{j}}{j !}=\left(1+n \frac{t}{N}\right)^{m}
$$

$$
P\left(Y_{m, m}>j\right)=\frac{(m)_{j}}{m^{j}},
$$

in agreement with Arnold's result.

\section{Acknowledgement}

The first author thanks Professor B. C. Arnold for his advice and help. 


\section{References}

[1] Arnold, B. C. (1972) The waiting time until first duplication. J. Appl. Prob. 9, 841-846.

[2] Comtet, L. (1972) Nombres de Stirling généraux et fonctions symetriques. C. R. Acad. Sci. Paris A 275, 747-750.

[3] COMTET, L. (1974) Advanced Combinatorics. D. Reidel, Dordrecht.

[4] MCCABE, B. (1979) Problem E. 2263. Amer. Math. Monthly 77, 1008. 